\title{
FLOATBOX: AN INNOVATIVE APPROACH TO DESIGNING METHODOLOGY FOR REMOTELY OPERATED UNDERWATER VEHICLE
}

\author{
Saurav Saha \\ Team Recon Subsea \\ SRM IST - KTR \\ Chennai, India
}

\author{
Mitransh Choubisa \\ Team Recon Subsea \\ SRM IST - KTR \\ Chennai, India
}

\author{
Rohan Tehalyani \\ Team Recon Subsea \\ SRM IST - KTR \\ Chennai, India
}

\author{
Aman Pandey \\ Team Recon Subsea \\ SRM IST - KTR \\ Chennai, India
}

\author{
Satyam Ambast \\ Team Recon Subsea \\ SRM IST - KTR \\ Chennai, India
}

\begin{abstract}
Working and stability of a remotely operated vehicle mainly depends on its structure and the buoyancy of the ROV. A ballast tank can be used to provide a dynamic buoyancy to the ROV. Dynamic buoyancy using a ballast tank provides stability and depth control to ROV but there is a drawback that the ballast tank consumes a significant amount of space when it comes to a relatively small ROV where size and space are extremely constrained. This (ballast tank) may not provide $100 \%$ water displacement and this may lead to the generation of a lot of drag force. This is eliminated by making a structure for the ROV which itself can serve as a ballast tank. This not only provides stability to the ROV but provides $100 \%$ water displacement. A structure can be constructed using PVC pipes which can be filled with water to make the ROV dynamically buoyant and the level of water inside the pipes can be controlled using a specialized Pneumatic System. Pneumatic buoyancy is a very popular phenomenon in the marine robotic field. Taking inspiration from that we are implementing a similar idea in ROV. This concept is very useful for small sized ROV. This can reduce the current consumption by the thrusters used for depth control.
\end{abstract}

Keywords- ROV, AUV, UUV, ballast tank, pneumatics, buoyancy

\section{INTRODUCTION}

With the recent development in the field of marine robotics, there is a need to find a solution for a dynamic buoyancy system. There has been a lot of work done in this area, but all that has been focusing on AUVs or UUVs. Thus, this paper focuses on a solution to the problem of ballast tank in small size ROVs. The goal is to introduce a technique which can modify the traditional structure which is used for small size ROVs. The current advancements in field of pneumatics and ballast tank can be combined and implemented together to find a structure which serve as both frame of a ROV as well as its ballast tank. The idea is to use high pressure air flow to control water level in the ballast tank.
This paper described the development of small size ROV frame which will also serve its ballast tank using the above described pneumatic system.

\section{Motivation}

The frame of the ROV should not only provide maximum amount of stability but also provide maximum amount water displacement in the waterbody. The work of the ballast tank is to provide dynamic buoyancy to the structure. The requirement of a dynamic buoyant system is very important because it can reduce a lot of current consumption during the vertical motion of the bot during a mission. The conventional way of providing buoyancy to the ROV is by using buoyancy foams and other buoyant materials. Buoyancy design and selection are critical to the successful operation of Remotely Operated Vehicles (ROVs). Low-density syntactic materials make ROVs neutrally buoyant, resistant to hydrostatic pressure and the ability to traverse to deeper depths for significantly longer \& extended periods of time. The above mechanism is usually implemented in an open box ROV structure. This is a very popular model used for providing maximum water displacement. This structure is built with a large flotation pack on top of an aluminum chassis to provide the necessary buoyancy to perform a variety of tasks. The sophistication of construction of the aluminum frame varies depending on the manufacturer's design. The size of structure and weight of aluminum usually make a standard ROV negatively buoyant and thus materials like foam boards, called buoyancy foams are used to make the ROV neutrally buoyant. Now the traditional methodology should be changed because of the fact that small size ROVs require maximum amount of water displacement and positioning of buoyancy foams alongside the electronics cabinet on the top of the ROV structure does not allow this. The drag force is also increased in the upward motion of the ROV which directly results in increased current consumption of thrusters. The ROV uses T100 and T200 thrusters, developed by Blue Robotics [11], for its motion. The upward motion is dependent on the T200 thrusters because of its ability to provide a greater amount of thrust and the fact that maximum drag force is required only during the upward and downward motion. In a 


\section{International Journal of Engineering Applied Sciences and Technology, 2020 \\ Vol. 4, Issue 12, ISSN No. 2455-2143, Pages 96-101 \\ Published Online April 2020 in IJEAST (http://www.ijeast.com)}

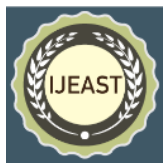

typical ROV design, buoyancy foam and electronics cabinet are designed to be kept on the top half of the structure. Thus, the thrusters are responsible for the vertical axis motion must be powerful enough to counter the drag force experienced by the structure. The combined weight of electronics cabinet and buoyancy foam is equal to $1.4 \mathrm{kgf}$. Now according to the data available the PWM value to counter the drag force is 1256 , at this value of PWM the current drawn will be 5.32 AMPs. Hence, a lot of current is required when all the six thrusters during its operation in a mission-based scenario.

All this data corresponds to this situation where the ROV is provided with a $12 \mathrm{~V}$ power supply from the top side control station.

The current consumption can be reduced easily with a modified frame structure with maximum water displacement which controls the amount of water in the ballast tank for depth control.

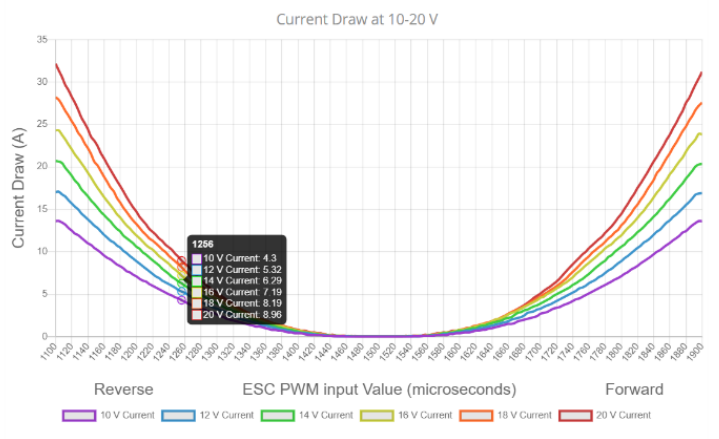

Fig. 1 Current v/s ESC PWM plot [11]

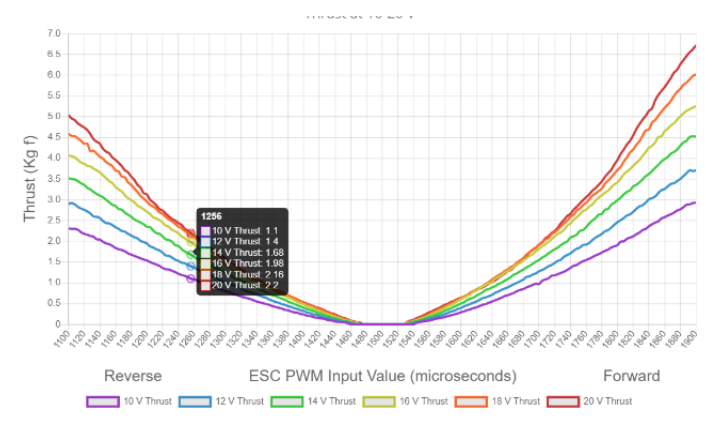

Fig. 2 Thrust v/s ESC PWM plot [11]

\section{RELATED WORK}

The need is to restore neutral buoyancy and to reduce the drag force. There has been a lot of tests conducted to calculate the water resistance on the structure of the ROV.[4] The test results show the importance of an open structure and how it reduces the drag force. This paper also focuses on the design and development of ballast tank. There has been a lot of work done in this field. The need of a ballast tank in a ROV is to control the buoyancy of the structure. ROVs use a lot of system for ballast tank. A ROV developed at the Massachusetts Institute of
Technology et al. (2003)," JAWS" [7], has major parts of the system as following, the air source, the manifold and solenoids used as the control system for the air, the pneumatic grippers, and the buoyancy control ballast tank. Plastic tubing is used as air lines between the major components. The source of the air is a surface tank. Another similar study is conducted by Anyuan Bi and Zhengping Feng et al. (2018) [9] where they developed a variable ballast tank system to change the total weight of their underwater vehicle. Another one such application of ballast tank to control an underwater robot is "NO MAD" et al. (1997) [10] which is an intelligent subsea robot that uses ballast tank for depth control. Variable buoyancy is also used for underwater gliders.[5] "ROBEX" et al. (2016) is a University of Bremen based project which uses pneumatic variable buoyance engine. They concluded that it appeared that the quickest way to build a buoyancy engine is to use a pneumatic concept. Not only ROVs but variable ballast tank has been fruitful in UUVs and AUVs as well. A DC-motor driven ballast tank was designed at National University of Singapore et al. (2013) [1] for their spherical underwater robot. The AUV "Theseus" et al. (2012) [8] has two air compressors for their two ballast tanks to control its buoyancy. There are a lot of examples where an external ballast tank is used to control the buoyancy of the structure, so this paper focuses on how the structure can be converted into a ballast tank. The controlling of this pneumatic system is done in the same way as ROV will be controlled. The solenoid valves to be used to control the flow of air will be given signals using Arduino [3].

\section{DESIGN AND DEVELOPMENT}

When the frame of an ROV is designed, there are two main aspects that must be decided upon. Firstly, it must provide maximum water displacement and secondly, the structure must be buoyant. The traditional idea of making a box like aluminum frame is proven to be inefficient. If we observe a typical model of an ROV which is widely used in marine industry, it is an aluminum frame with a lot of holes in it to let the water pass through it. The reason for this is that aluminum is comparatively light weight when considering other metals. This model makes the ROV negatively buoyant eventually and thus buoyancy foams come into play. This problem of finding a light weight material with buoyancy can be eliminated by a common daily life simulation. Visualize dragging a cube and a round object, clearly the drag force is more in case of a cube or say a cuboidal surface. The same principle applies underwater too.

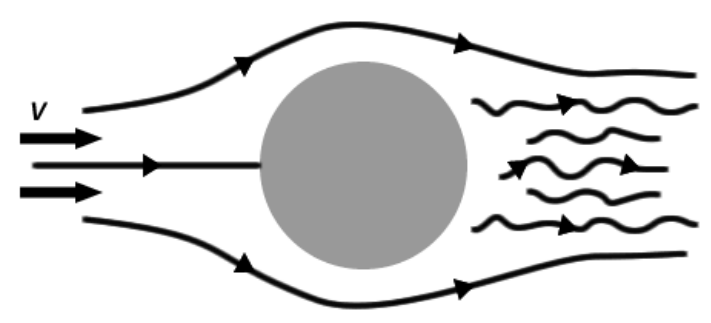

Fig. 3 Water flow around sphere 
Water resistance is a concept almost similar to air resistance as both of them are fluids. The water resistance that is experienced is

clearly more in case of a cube/cuboidal structure compared to that of a sphere or a streamlined body. This concept is used for finding an appropriate material for the construction of the frame of an ROV which is both stable and strong. After a lot of simulations and testing a variety of materials, the best suited material for a frame which serves both as structure and the ballast tank, turned out to be a Polyvinyl Chloride (PVC) in the form of pipes. The cylindrical structure of pipe has a drag coefficient of 0.18 which is least whereas the drag coefficient of that of a cube is 2.3.[2] This pipe like structure has been used because the volume of water in the cylindrical cavities of the structure can be manipulated using a general pneumatic system. Usage of PVC also significantly decreases the cost of fabrication of the ROV.

\section{A. Frame and Structure}

After the selecting the material for construction of the structure, the next step is to design a durable and steady frame and see how the concept of a ballast tank can be implemented on it. A lot of designs were tried and tested and finally the conclusion was to create two square-like plane structures using PVC pipes and joining both of them from their mid-points. All the PVC pipes are joined using T-connectors or L-connectors. The idea of making a square structure is to increase the stability and strength. The use of PVC pipes for making the structure of the ROV is not a new thing, many small sized ROVs are made using PVC pipes, but the same idea can be implemented with some innovative modifications. The most common use of PVC pipes is for making a cube or cuboidal shaped structure. This design also has the same objective to allow maximum displacement of water plus when small scale ROVs are made, cost of material is also a significant factor, PVC pipes serve for exactly that purpose. The other very important advantage is that PVC is easily available. The requirement is to construct a ballast tank-based structure where 3 -inch pipes can be used. Different sizes of pipes were considered and this size was best suited based on our application because it is both durable and has enough volume to store the water at a required level. The structure as described above, two squares connected with one pipe is best suited for our requirements and thruster positioning. A network of pneumatic pipes is also set alongside the PVC pipes for balancing the water level and air pressure. There is one pneumatic pipe running alongside the power cable and ethernet cable in the tether to control the air pressure from the dock area. The pictures below elaborately describe the structure explained previously. It shows how the use of PVC pipes allows the ROV to handle water resistance in a significantly more efficient manner. This unique feature of the frame allows us to place the thrusters in such a manner so as to provide maximum water displacement. This will be explained in further sections. The thrusters can be placed on the pipes with the electronics cabinet on the top of the structure. The placing of thrusters' mounts on the PVC pipes is done by using rivets. Rivets are also used for connecting all the pipes and their corresponding connectors. This adds to the rigidity of the structure. Considering that the material to be used to fabricate the structure of the ROV uses PVC pipes, it is crucial to add an additional mechanism to ensure the rigidity of structure. This is the major difference between a typical ROV and the proposed structure.
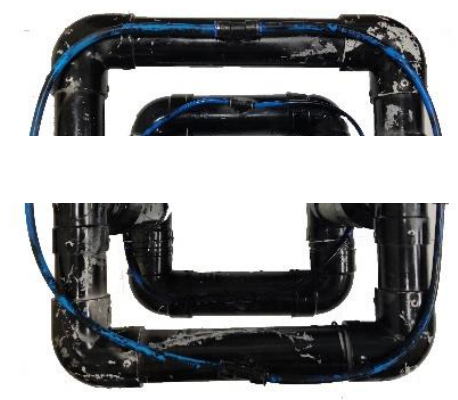

Fig. 5 Top view of structure

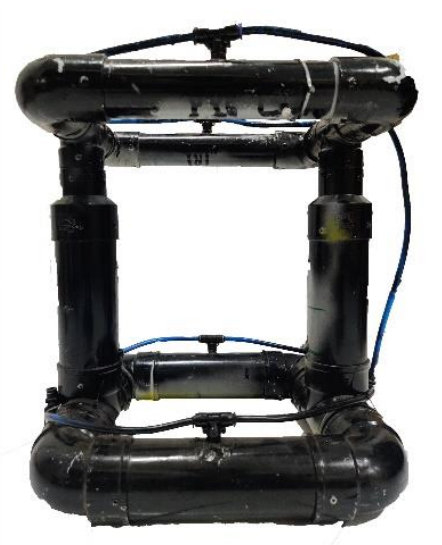

Fig. 4 Front view of structure

\section{B. Thruster positioning}

The placement of thrusters is done on the basis of analysing the response of the structure with respect to the fluid and the positioning is ascertained by testing the water displacement that is observed. According to our requirement, we need in total of six thrusters. The usual requirement of the positioning of the thruster is to provide the maximum degrees of freedom. The design of our bot can have two lateral degrees of freedom in $\mathrm{x}$ and $y$ and one rotational degree of freedom around y-axis. It has a lateral degree of freedom on $\mathrm{z}$-axis too, apart from this there is a rotational degree of freedom around $z$-axis, this gives in total of five degrees of freedom to the ROV. The positioning of thrusters is shown in the following diagram. The thrusters 


\section{International Journal of Engineering Applied Sciences and Technology, 2020 \\ Vol. 4, Issue 12, ISSN No. 2455-2143, Pages 96-101 \\ Published Online April 2020 in IJEAST (http://www.ijeast.com)}

illustrated using blue color are the ones employing a clockwise direction and the ones in green move in a clockwise direction.

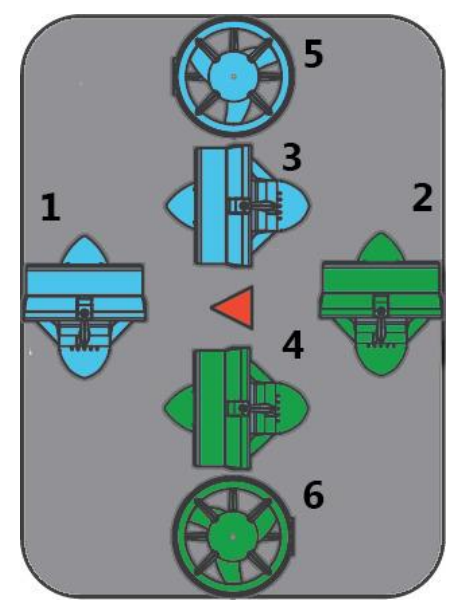

Fig. 6 Thruster position

When two thrusters of clockwise and counter clockwise direction are run together then a forward motion is possible. The red triangle signifies the front side of the ROV. Thruster 3 and 4 are responsible for forward and backward motion, 1 and 2 are responsible for left and right motion as well as a rotational motion around $\mathrm{z}$-axis, which can also be accessed by thruster numbers 3 and 4 . Finally thruster 5 and 6 are responsible for motion in the z-axis, i.e., depth adjustments, other than this it can also provide a rotational motion around y-axis.

The positioning of thrusters on the PVC pipes is done by placing them on customized 3D printed mounts which are them fixed on the pipes with the help of rivets.

The above picture shows us the right-hand side view of the ROV frame, where we can observe the positioning of thruster number 1, 2 and 5 in it. The clear view of thruster arrangements on the frame can be seen in following figures.

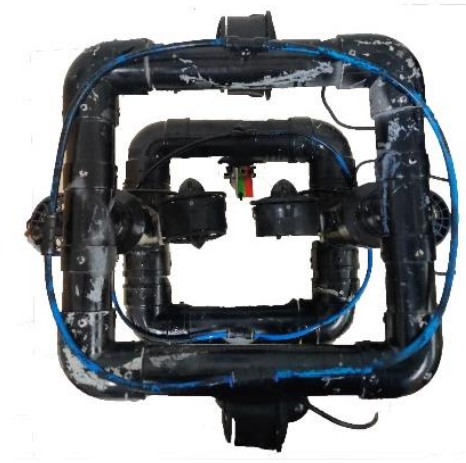

Fig. 8 Top view of thruster position

The length of pipes in upper square is $37 \mathrm{~cm} \times 37 \mathrm{~cm}$, and the size of inner square on top side is $20 \mathrm{~cm} \times 20 \mathrm{~cm}$. The length of pipes

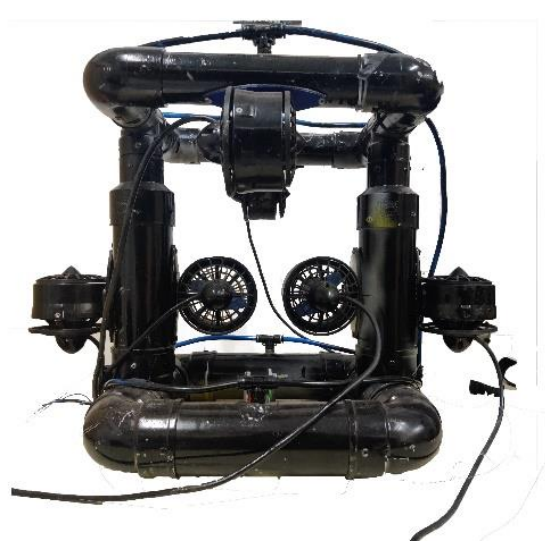

Fig. 7 Front view of thruster positioning

on bottom side of frame is $40 \mathrm{~cm} \times 40 \mathrm{~cm}$ and the inner square on bottom side is $17 \mathrm{~cm} \times 17 \mathrm{~cm}$. The size of the bottom square is not in symmetry with the upper square, this is done to maintain stability of structure in water. When this structure is immersed in the water, water starts to enter the bottom square. It prevents it from experiencing an upside-down torque inside the water which can cause it to flip. This was tried and tested with different combinations of dimensions of the upper and lower square.

\section{Pneumatic system}

The frame of ROV is described in the preceding section, but the objective is to study the working of ballast tank. The notion of pipe structure to be used as ballast tank is self-explanatory by the illustrations shown in preceding sections. This section explains the working mechanism of the structure in conjunction with the ballast tank. A network of pneumatic pipes is laid alongside the PVC pipes. These pneumatic pipes are connected via a $\mathrm{T}$ connectors and the size of pipes are $6 \mathrm{~mm}$ and $4 \mathrm{~mm}$. The air pressure is controlled by a compressor on the shore and a pressure valve is installed to monitor the pressure level. There is an inlet hole in the bottom square of the structure for the water

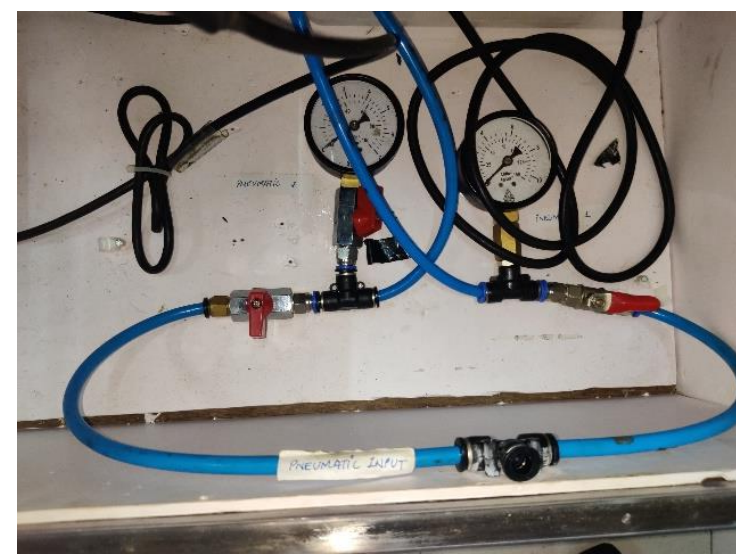

Fig. 9 Pneumatic network and pressure gauge 


\section{International Journal of Engineering Applied Sciences and Technology, 2020 \\ Vol. 4, Issue 12, ISSN No. 2455-2143, Pages 96-101 \\ Published Online April 2020 in IJEAST (http://www.ijeast.com)}

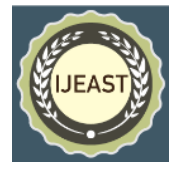

to enter inside the pipes. Once the water is inside the pipes, its level can be adjusted according to depth requirements. A pressure of $0.56 \mathrm{~kg} / \mathrm{cm}^{2}$ to $0.84 \mathrm{~kg} / \mathrm{cm}^{2}$ is applied by the compressor on the shore.

\section{RESUlT AND DisCUSSION}

The dynamic buoyancy control in the ROV is maintained with the use of a pneumatic system which regulates air supply in the ballast tank made of PVC pipes, which itself serve as the frame for ROV. The structure is positively buoyant when immersed into the water initially, as soon as the water starts entering from the inlet of the PVC pipes, it gradually submerges into the water. The maximum amount of water which the structure can hold turned out to be 10 litres and at this stage the structure becomes negatively buoyant. The air pressure inside the ballast tank is continuously monitored and maintained at a particular amount to control the amount of water inside the tank at all times with the proper calibration of the thrusters to a particular PWM value provides the ROV with accurate depth control. After the usage of water for depth control, the thrusters are now run to maintain the depth. The PWM value required to run the thrusters is now changed from 1256 to a range of 14501280(counter clockwise) and 1550-1700(clockwise) (all tests done at $12 \mathrm{~V}$ DC supply). The current usage by the thrusters is in the range of 0.1 AMPs to 4 AMPs. A fuse of 5 AMPs is additionally installed to ensure safety and to not allow the current to exceed the required threshold during functioning. To maintain a particular depth level the PWM value required is 1550. At this value of PWM, the pulse is sent to the thrusters after a certain delay. The thrusters only run on particular time intervals to maintain the depth of the ROV at a particular level. This reduces the current usage significantly.

\section{CONCLUSION}

The structure of pipes turns out to be quite light weight but at the same time very rigid for stability. The buoyancy is adjustable. For the future the same idea can be implemented in commercial ROVs with the help of proper enclosures. The pneumatic system can also be used to pressurise the electronics cabinet and to use piston-based manipulators, thus the same network can be multipurpose. To reduce the tether load, on board pumping mechanism is an efficient option. The low-cost small size ROVs, AUVs and UUVs can feasibly be manufactured using the proposed work.

\section{ACKNOWLEDGMENT}

The authors would like to thank Dr. A Vimala Juliet, Professor and Head, Dept of EIE, SRM Institute of Science and Technology, for allowing the team to conduct its research under her department. We would also like to thank Mr. J Sam Jeba Kumar, Assistant Professor, Dept of EIE, SRM Institute of Science and Technology for his guidance and support to the team through the project. The team would finally like to acknowledge SRM Institute of Science and Technology for giving it the space and resources to conduct its project.

\section{REFERENCE}

[1] A. Agrawal, B. Prasad, V. Viswanathan and S. K. Panda,(2013). "Dynamic modelling of variable ballast tank for spherical underwater robot," IEEE International Conference on Industrial Technology (ICIT), Cape Town, 2013, pp. 58-63.

[2] J. Sahili, A. E. Hamoud and A. Jammoul, (2018). "ROV Design Optimization: Effect on Stability and Drag force," 2018 6th RSI International Conference on Robotics and Mechatronics (IcRoM), Tehran, Iran, pp. 413-417

[3] A. Ghilezan and M. Hnatiuc,( 2017). "The ROV communication and control," 2017 IEEE 23rd International Symposium for Design and Technology in Electronic Packaging (SIITME), Constanta, pp. 336-339. doi: 10.1109/SIITME.2017.8259920

[4] S. Fan, L. Lian, P. Ren and L. Ao,( 2012). "Resistance calculation and motion simulation for deep sea openframed remotely operated vehicle based on hydrodynamics test," 2012 Oceans - Yeosu, Yeosu, pp. 1-5.

[5] C. Waldmann, T. M. Kier, W. S. Kirkwood and S. Meckel,( 2016). "Performance data of a pneumatic variable buoyancy engine for a newly designed underwater glider," OCEANS 2016 MTS/IEEE Monterey, Monterey, CA, pp. $1-3$..

[6] S. B. Pranesh, D. Sathianarayanan, E. Chandrasekar, M. Murugesan, G. A. Ramadass and M. A. Atmanand, (2015). "Design and development of polypropylene frame for remotely operated vehicle," OCEANS 2015 - Genova, Genoa, pp. 1-6.

[7] K. S. Wasserman, J. L. Mathieu, M. I. Wolf, A. Hathi, S. E. Fried and A. K. Baker,( 2003). "Dynamic buoyancy control of an ROV using a variable ballast tank," Oceans 2003. Celebrating the Past ... Teaming Toward the Future (IEEE Cat. No.03CH37492), San Diego, CA, USA, pp. SP2888-SP2893 Vol.5.

[8] S. A. Woods, R. J. Bauer and M. L. Seto,( Oct. 2012). "Automated Ballast Tank Control System for Autonomous Underwater Vehicles," in IEEE Journal of Oceanic Engineering, vol. 37, no. 4, pp. 727-739.

[9] A. Bi and Z. Feng,( 2018). "Hierarchical Control of Underwater Vehicle Variable Ballast Systems," 2018 37th Chinese Control Conference (CCC), Wuhan, pp. 39113914.

[10]Zhongqun Lu, M. Hinchey and D. Friis,( 1997). "Development of a small pneumatic subsea robot," CCECE '97. Canadian Conference on Electrical and Computer Engineering. Engineering Innovation: Voyage of 
Discovery. Conference Proceedings, Saint Johns, Newfoundland, Canada, pp. 442-445 vol.2.4

[11] Blue Robotics. T200 technical details. Retrived from https://bluerobotics.com/store/thrusters/t100-t200thrusters/t200-thruster/

[12] Xiaole $\mathrm{Xu}, \&$ Canjun Yang. (2015). A pneumatic-driven airdropped portable underwater profiler for rapid deployment. OCEANS 2015 - MTS/IEEE Washington. doi:10.23919/oceans.2015.7404403 